\title{
New Perspectives on the Diagnosis and Management of Duchenne Muscular Dystrophy
}

\section{Proceedings of a Symposium Presented at the 13th International Congress on Neuromuscular Diseases 2014 on 10 July in Nice, France}

\author{
Expert reviewed by:
}

Symposium Speakers: Francesco Muntoni, ${ }^{1}$ Annemieke Aartsma-Rus, ${ }^{2,3}$

Eugenio Mercuri ${ }^{4}$ and Hanns Lochmüller ${ }^{3}$

\begin{abstract}
1. The Dubowitz Neuromuscular Centre, University College London, London, UK; 2. Leiden University Medical Center, Leiden, The Netherlands; 3. John Walton Muscular Dystrophy Research Centre, Institute of Genetic Medicine, Newcastle University, Newcastle, UK; 4. Catholic University of the Sacred Heart, Rome, Italy
\end{abstract}

\begin{abstract}
Duchenne muscular dystrophy (DMD) is a rare X-linked recessive disorder that occurs in around one in 5,000 male births. The prevalence of DMD is expected to rise due to improved standards of care and implementation of guidelines, leading to longer survival. Specialist genetic confirmation of a DMD diagnosis is typically followed by access to specialist care and treatment: the exact DMD-causing mutation should be identified because it can influence prognosis and identify patients eligible for treatment. Since the majority of patients has a deletion or duplication of one or more exons $(\sim 70 \%)$, generally multiplex ligation-dependent probe amplification suffices to identify the mutation. Exon sequencing is performed to pick up small mutations ( 30\%). Greater awareness of DMD is needed among healthcare professionals to enable earlier diagnosis, which would facilitate family planning, as well as patient care and treatment. In DMD patients who are still able to walk, the 6-minute walk test (6MWT) has been shown to be a valid measure of physical functioning and a predictor of disease progression, with high inter-test reliability. In a study of the natural history of DMD, change in 6MWT of around 30 metres has been indicated to be clinically relevant and clinically meaningful. DMD patients responded to treatment as shown by the improvement in the 6MWT score in the large multinational trial of the nonsense mutation readthrough agent, ataluren (Translarna ${ }^{\mathrm{TM}}$ ) $40 \mathrm{mg} / \mathrm{kg} / \mathrm{day}$, where treatment was associated with a 31.3 metres improvement on the 6MWT distance, after 48 weeks, compared with placebo. The Translational Research in Europe-Assessment \& Treatment of Neuromuscular Diseases (TREAT-NMD) network was launched to provide an infrastructure to accelerate research and therapy development, increasing collaboration, improving patient care and helping to support 'clinical trial readiness'. As such, the TREAT-NMD registry network is well placed to support further understanding of DMD and the impact of therapies that may be used over the long term, permitting a host of research questions to be explored.
\end{abstract}

\section{Keywords}

6-minute walk test (6MWT), ataluren, clinical outcome measures, Duchenne muscular dystrophy (DMD), genetic testing, nonsense mutation, Translarna ${ }^{\text {TM }}$, Translational Research in Europe-Assessment \& Treatment of Neuromuscular Diseases (TREAT-NMD), diagnosis

\footnotetext{
Disclosures: Francesco Muntoni is employed by UCL. He is the principal investigator of the PTC Therapeutics Inc.-sponsored phase III trial on Translarna, the Prosensasponsored PRO045 and PRO051, of the Sarepta Therapeutics Inc.-sponsored 4053 trial and of the Summit PLC-sponsored trial for C1100. He has been a member of Pfizer scientific advisor board since 2014. In the past three years he has provided ad hoc consultancies for PTC Therapeutics Inc., Sarepta Therapeutics Inc. and Summit PLC. Annemieke Aartsma-Rus is employed by LUMC, which has patents on exon skipping technology. As a co-inventor, Annemieke Aartsma-Rus is entitled to a share of royalties. She also works as an ad hoc consultant for Global Guidepoint, in which case fees are paid to LUMC. Eugenio Mercuri has served on clinical steering committees and/or as a consultant for Eli Lilly, Italfarmaco, Prosensa, PTC Therapeutics and Shire. Hanns Lochmüller has no conflicts of interest in terms of Duchenne muscular dystrophy work. Acknowledgements: Editorial assistance was provided by Catherine Amey of Touch Medical Media, London, UK. This article reports the proceedings of a sponsored satellite symposium and as such has not been subject to the journal's usual peer-review process.

Open Access: This article is published under the Creative Commons Attribution Noncommercial License, which permits any non-commercial use, distribution, adaptation and reproduction provided the original author(s) and source are given appropriate credit.

Received: 18 December 2014 Accepted: 26 January 2015 European Neurological Review, 2015;10(1):73-8 DOI: 10.17925/ENR.2015.10.01.73

Correspondence: Francesco Muntoni, The Dubowitz Neuromuscular Centre, University College London, London, WC1N 1EH, UK. E: f.muntoni@ucl.ac.uk
}

Support: The publication of this article and the $13^{\text {th }}$ International Congress on Neuromuscular Diseases satellite symposium that it reports were supported by PTC Therapeutics, Inc. The views and opinions expressed are those of the presenters and not necessarily those of PTC Therapeutics, Inc. 


\title{
Duchenne Muscular Dystrophy - Introduction
}

\author{
Francesco Muntoni
}

The Dubowitz Neuromuscular Centre, University College London, London

\begin{abstract}
Duchenne muscular dystrophy (DMD) is a rare X-linked recessive disorder mutation in the DMD gene, Xp21. It is a progressive disease that is characterised by lack of dystrophin, which leads to severe myofibre degeneration. This, in turn, results in a relentless decline in physical functioning with subsequent respiratory and cardiac failure, leading to early death. One estimate of cost of DMD from a recent study carried out by Translational Research in EuropeAssessment \& Treatment of Neuromuscular Diseases (TREAT-NMD) and GSK using TREAT-NMD registries in four countries found the yearly society cost to be in the region of $\$ 80,120-120,910(€ 70,765-106,792)$ per patient and the corresponding household burden between $\$ 58,440-71,900$ ( $€ 51,616-$ 63,505). ${ }^{1}$ However, due to difficulties in capturing end-of-life costs for DMD patients, these may be conservative estimates.
\end{abstract}

Recent estimates put the birth incidence in males of DMD at approximately $1: 5,000 .{ }^{2,3}$ The natural history of DMD is becoming better understood and care guidelines are available that help physicians provide optimal care. The global prevalence is expected to rise due to improved survival and standards of care. These improvements are the result of better understanding of DMD complications, implementation of guidelines and enhanced care practices, for example, the introduction of non-invasive positive pressure ventilation (NIPPV) for respiratory complications. ${ }^{4}$
Kaplan-Meier survival analyses from the Newcastle Muscle Centre, International Centre for Life, Newcastle upon Tyne, UK showed significant decade on decade improvement in survival: the mean age of death in the 1960s was 14.4 years, whereas for those who had received NIPPV since 1990, it was 25.3 years. ${ }^{5}$ Current unpublished figures from the London neuromuscular centre - in collaboration with Professor Anita Simonds (Brompton Hospital), place the current survival of NIPPV patients at 29 years (Simonds et al., personal observation).

Another important advance is the use of glucocorticoid therapy to improve muscle strength and function. ${ }^{6,7}$ In an assessment of 360 boys aged 3-15 years who were treated with daily or intermittent ( 10 days on/10 days off) prednisolone for a mean duration of treatment of 4 years, the median loss of ambulation was 12 years in the intermittent treatment group and 14.5 years in the daily treatment group. ${ }^{8}$ Despite these advances in standards of care and the emerging understanding of DMD natural history, progressive muscle loss in DMD cannot currently be avoided, and loss of ambulation in patients with DMD is eventually followed by loss of upper limb function, loss of self-feeding and respiratory insufficiency. Restoring dystrophin expression remains the ultimate goal for treatment so that the time to these events could be delayed as much as possible.

\section{Diagnosing Duchenne Muscular Dystrophy in the Era of Mutation-specific Therapies}

\author{
Annemieke Aartsma-Rus \\ Leiden University Medical Center, Leiden, The Netherlands, \\ John Walton Muscular Dystrophy Research Centre, Institute of Genetic Medicine, Newcastle University, Newcastle, UK
}

Dystrophin acts as a shock absorber by connecting cytoskeletal actin within muscle fibres to the extracellular matrix surrounding muscle fibres. In DMD, dystrophin is prematurely truncated and non-functional. This contrasts to Becker Muscular Dystrophy (BMD), which is typically caused by in-frame dystrophin deletions that allow the production of an internally deleted but partially functional dystrophin. BMD occurs in approximately one in 20,000 men.

Deletions or duplications of one or more exons are the most common mutation in DMD accounting for over two-thirds of DMD cases. ${ }^{9}$ Small mutations have also been described, including nonsense mutations, small insertions, deletions, inversions and splice site mutations. Other rarer mutations found include intronic mutations and pseudoexon activation, translocations and missense mutations.

A deletion or duplication disrupts the reading frame for DMD, i.e. the number of nucleotides missing (or being duplicated) from the exons is not divisible by three, resulting in a shift in the translational reading frame, and aberrant amino acids used for protein formation and generally a premature truncation signal. Similarly, small insertions/deletions can also disrupt the reading frame. For splice site mutations the exon is not recognised and excluded from messenger RNA (mRNA), which (like deletions) can lead to a reading frame shift if the number of nucleotides in that exon is not divisible by three. In the case of nonsense mutations, which affect approximately one in 27,000 male births ( $13 \%$ of mutations), the reading frame is not affected, but a codon for an amino acid is changed into a stop codon, causing the dystrophin protein to truncate prematurely and being non-functional.

The frequency of the various mutations in males with DMD were estimated using data from the Leiden Open (source) Variation Database (LOVD; $n=4,770)^{9}$ and the TREAT-NMD Global database $(n=7,172) .{ }^{10}$ Deletions were the most common mutations identified and were present in $65 \%$ of patients in the LOVD database and $69 \%$ in the TREAT-NMD registry. The location and extent of the in-frame mutation was found to impact on the disease severity of Becker patents, as shown in Figure 1.9.11 When a deletion disrupts the crucial actin-binding domains and/or the binding domain for beta-dystroglycan (which connects to the extracellular matrix) the resulting dystrophin is not functional and patients have a DMD phenotype. Also, when there is a large deletion in the central domain (>36 exons), a DMD phenotype will generally result. Frame-shifting dystrophin mutations would be expected to result in DMD, but mutations prior to exon 8 can present as BMD instead as there are alternative start sites in exon 8 , and this can compensate for the earlier mutation. Furthermore, frame-shifting mutations flanking exon 44 generally have a more mild disease course than typical DMD. 
Figure 1: In-frame Mutations and Dystrophin Phenotypes

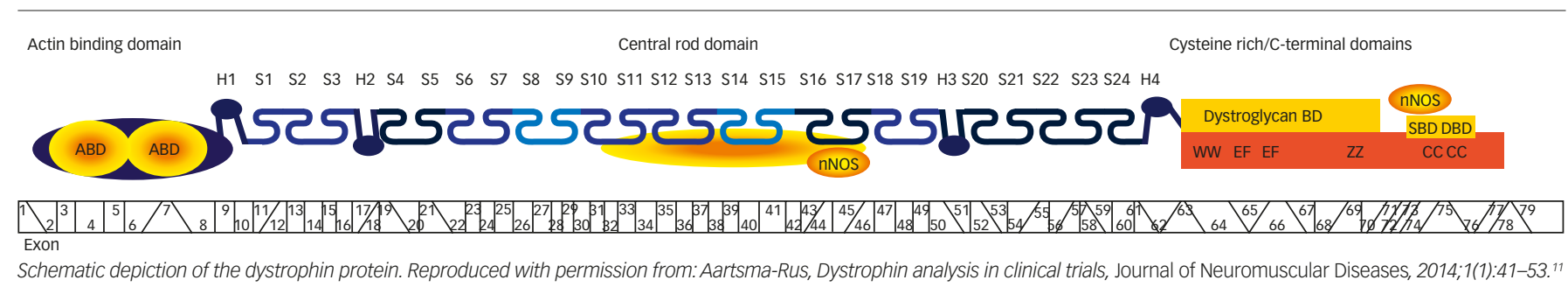

Early diagnosis is crucial for DMD to allow informed family planning and also appropriate planning for the patient's care and treatment. ${ }^{12,13}$ Medical examination should be carried out by a neuromuscular disease specialist and should be considered when: the child is male, muscle function is impaired (as revealed by frequent falls, Gower's sign, etc.), there is delayed speech (which may be more pronounced than muscle impairment), serum creatine kinase levels are elevated (10-100 times normal) accompanied by high aspartate transaminase and alanine transaminase levels. ${ }^{12,13}$ Genetic confirmation, requiring full complementary DNA (CDNA) sequencing in patients in whom no deletion or duplication can be found, is essential to allow for carrier testing and to find out whether the patient is eligible for mutation-specific approaches to DMD management.

Several methods are required for genetic confirmation of DMD. Multiplex polymerase chain reaction (PCR) generally uses two sets of primers to detect the presence or absence of a selected number of exons. The method is inexpensive and straightforward but for many patients the exact extent of the deletion is not known, while this is crucial to assess if the reading frame is disrupted or maintained. Also, this method does not detect duplications and cannot be used for carrier detection. Multiplex ligation-dependent probe amplification (MLPA) detects the abundance of each DMD exon and can therefore be used to exactly pinpoint deletions as well as duplications in patients and carriers. Given that deletions and duplications of one or more exons occur in $>70 \%$ of patients, MLPA will pick up the majority of mutations. When MLPA does not reveal a deletion or duplication in DMD, each exon of the dystrophin should be analysed by Sander sequencing, to provide a genetic diagnosis for DMD. Eventually, whole exome sequencing may replace MLPA and exon analysis.

Generally muscle biopsies are not needed for diagnosing DMD. A muscle biopsy allows protein analysis by Western blotand/or immunohistochemistry to reveal the size and abundance of dystrophin, and RNA analysis to, for example, test for the inclusion of cryptic exons. When protein analysis shows the absence of dystrophin, however, DNA analysis is still necessary. By contrast, if a DNA mutation has been found, a biopsy is not generally needed, with the possible exception of discordant phenotypes, whereby a DMD phenotype is expected but BMD develops or vice versa.

In a retrospective analysis in Newcastle over 10 years, the age of diagnosis fell by a disappointingly small increment, from 4.5 to 4.1 years. ${ }^{14}$ This is likely a reflection not of the inability to detect a mutation but in the delay to engage a healthcare professional. In this dataset $(n=20)$, the first symptoms of DMD became apparent at 2.7 years of age; however, a healthcare professional was not engaged typically until a patient was around 3.6 years old. Creatine kinase levels were measured at 4.2 years and confirmation of diagnosis occurred at 4.3 years of age. The diagnostic delay in this dataset was approximately 19 months although an estimate in the US of the diagnostic delay of DMD was as high as 30 months. More awareness of DMD among general practitioners is therefore crucial to allow earlier diagnosis and efforts to develop neonatal screening for DMD are underway.

\title{
What is a Realistic Target Therapeutic Benefit for Dystrophin Muscular Dystrophy Patients and their Families?
}

\author{
Eugenio Mercuri
}

Catholic University of the Sacred Heart, Rome, Italy

\begin{abstract}
A functional outcome measure for DMD to be used to detect clinical benefit in clinical trials should, among other criteria, be reliable, validated with other measures, suitable for multicentre studies, yield normative ranges and natural history data and show sensitivity to changes/responsiveness. The 6-minute walk test (6MWT) is a commonly used outcome measure in ambulant boys with $\mathrm{DMD}_{1}^{15}$ is reliable and suitable for multicentre studies and has been selected as an outcome measure in DMD clinical trials and ongoing natural history studies. ${ }^{15-18}$ In the 6MWT, the distance covered walking fast as possible on flat ground for 6 minutes is recorded. A 25 metre course is marked by two cones at each end, around which the patient will turn. The patient can stop if tired, but not sit, and resume walking as soon as possible. Distances are marked at each lap and every minute.
\end{abstract}

The 6MWT has been shown to be feasible, safe and reproducible as an outcome measure for DMD natural history and therapeutic trials. ${ }^{15-18}$ A longitudinal study that was conducted to assess changes over 24 months in ambulant boys with DMD showed that there was an average overall decline of -22.7 (standard deviation [SD] 81.0) in the first year on the 6MWT and -64.7 (SD 123.1) in the second year.19 More recently, a 3-year study following up these 24-month data showed that age was an important determinant to prognosis in $\mathrm{DMD}$, with children older than 7 years of age showing a much more rapid deterioration on the 6MWT than children below the age of 7 years..$^{20}$ This follow-up study also demonstrated that baseline walking and functional ability, as measured by 6MWT performance, is also a major factor in determining the rate of DMD patient decline. These findings are consistent with other longitudinal natural history observations in DMD. ${ }^{15}$ 
Figure 2: Observed Mean Change in 6-Minute Walk Test with Ataluren (40 $\mathrm{mg} / \mathrm{kg} / \mathrm{day})$

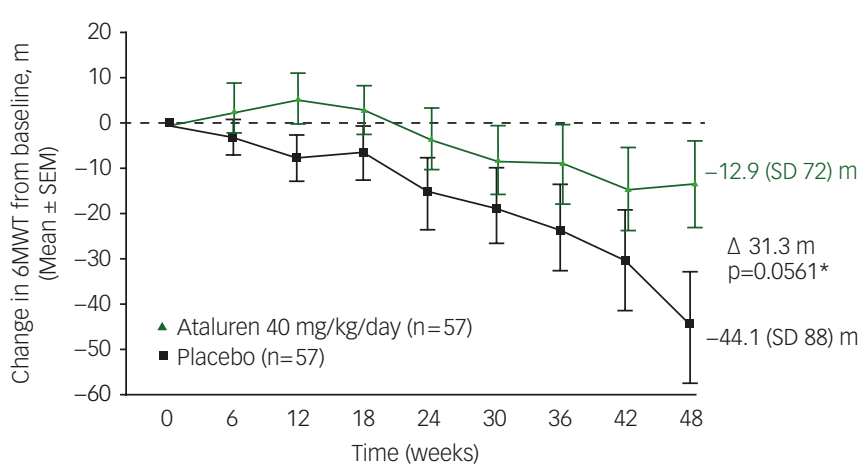

6MWT = 6-minute walk test; $S D=$ standard deviation; SEM = standard error of the mean

A decline of 30 metres on 6MWT has been demonstrated to be clinically meaningful and predictive of loss of ambulation and correlated with a clinically meaningful change in QoL. ${ }^{21}$ The 6MWT has been validated against other measures in DMD, including measures of muscle strength (knee extensors), ${ }^{22}$ gross motor skills (the functional scale, North Star Ambulatory Assessment [NSAA]), ${ }^{18,19}$ endurance (continuous step activity) ${ }^{16}$ and timed items (10 metres and Gowers). ${ }^{19}$ The NSAA is a clinical evaluation tool designed specifically for children with DMD. The NSAA protocol sets out a standard timed function test for clinicians to assess the ability of the child to perform 17 activities, including standing, head-raising, hopping and running. The NSAA is a practical and reliable test that can be completed in 10 minutes $^{23,24}$ and, with adequate training in place, can be used in a multicentric setting for DMD clinical research and trials. ${ }^{24,25}$

As previously stated, outcome measures in DMD should be sensitive to change and responsive to treatment. Based on two statistical distribution methods, the minimal clinically important differences (MCIDS) in DMD for the 6MWT have been reported to be 28.5 metres and 31.7 metres.22
In the phase IIb trial (sponsored by PTC Therapeutics, Inc.) of the nonsense mutation readthrough agent, ataluren (Translarna ${ }^{\mathrm{TM}}$ ), nonsense mutationmediated DMD patients receiving ataluren $(40 \mathrm{mg} / \mathrm{kg} /$ day) showed a clinically meaningful difference (approximately 30 metres) in the change in their 6MWT results compared with the placebo arm. Treatment resulted in a mean decline from baseline in 6MWT of 12.9 metres compared with 44.1 metres for patients receiving placebo over 48 weeks ( $p=0.0561$; see Figure 2), suggesting that the 6MWT measure is responsive to treatment in ambulatory DMD patients. ${ }^{26}$

The changes in 6MWT have also been correlated with timed function tests in DMD, such as the 10 metre walk/run. ${ }^{22}$ In addition, 6MWT changes are associated with changes in other measures reflecting other aspects of daily activities, e.g. the functional scale, NSAA. ${ }^{19,27}$ The $6 \mathrm{MWT}$ has also been correlated with person-reported outcomes (Paediatric Outcomes Data Collection Instrument [PODCI). ${ }^{19}$

It is also important to develop outcome measures for use in younger children, although this can be challenging. In a study by De Sanctis et al. ${ }^{28}$ including 147 assessments in normally developing boys and 144 assessments in boys with DMD, who were aged between 3 and 5 years, the NSAA appeared to be sensitive and suitable in DMD patients of 4 years and older. However, further research is ongoing in scales that may be useful in DMD patients as young as 3 years. ${ }^{29,30}$

Assessment of upper limb function presents another challenge and this is particularly important both in non-ambulatory DMD patients and to support a consistent measure across the spectrum of DMD. Efforts to develop such reliable and valid upper limb assessment tools across the dimensions of the shoulder, elbow and wrist/finger are currently ongoing. ${ }^{31,32}$ The aim will be to cross-validate these tools with other measures including mytools (grip strength, key pinch, and repetitive flexion/extension movements of wrist and fingers (MoviPlate) and magnetic resonance imaging (MRI)

\title{
Understanding Long-term Outcomes in Duchenne Muscular Dystrophy
}

\author{
Hanns Lochmüller
}

John Walton Muscular Dystrophy Research Centre, Institute of Genetic Medicine, Newcastle University, Newcastle, UK

The need for long-term outcome data in DMD is being met from diverse sources such as patient registries, natural history studies, post-marketing surveillance and clinical trials. Data quality, accuracy and completeness are the highest in randomised controlled trials but generating these with sufficient patient numbers is difficult in such a rare disease and the cost per patient is high. Long-term outcome data with patient registries have high patient numbers, and may be generated from patient registries although the data completeness and data/quality accuracy tends to be lower than clinical trials.

Recent years have seen rapid developments in the neuromuscular field and, in January 2007, the TREAT-NMD network was launched to unite stakeholders-including patients, advocacy groups, clinicians, researchers, biomedical industry experts and government agencies - by providing an infrastructure to accelerate research and therapy development, increasing collaboration, improving patient care and helping to support 'clinical trial readiness'. 33,34 TREAT-NMD was initially established as a EUfunded network of excellence with the remit of reshaping the research environment of the neuromuscular field. The TREAT-NMD network has since expanded from its European roots to become a global organisation as TREAT-NMD Alliance.

In the TREAT-NMD network, genetic and clinical data have been standardised to generate maximum uptake and a minimal dataset for trial recruitment. Standardised items agreed for inclusion in TREATNMD include personal patient data (name, date of birth, address); diagnosis (DMD, BMD, female carrier); presence and type of mutation or deletion; motor function (ambulation/wheelchair use); medication use (steroids); scoliosis surgery as well as family history; cardiomyopathy, echocardiography results (left-ventricular ejection fraction); ventilator function, forced vital capacity, ventilator use, and muscle biopsy.

The TREAT-NMD network adheres, via the TREAT-NMD registry charter, to the principles of providing patients with feedback, the possibility of data withdrawal, an informed consent form, pseudonymised data and frequent data updates. Currently, the registry includes more than 10,000 


\section{Figure 3: Duchenne Muscular Dystrophy Exon Skipping - Data from Registries for Trial Feasibility and Recruitment}

\begin{tabular}{|l|l|l|l|l|l|l|}
\hline & Exon 44 & Exon 45 & Exon 50 & Exon 51 & Exon 53 & all \\
\hline $\begin{array}{l}\text { Belgium } \\
\text { Bulgaria }\end{array}$ & 11 & 18 & 11 & 36 & 14 & 90 \\
\hline $\begin{array}{l}\text { Czech Republic } \\
\text { and Slovakia }\end{array}$ & 8 & 3 & 0 & 2 & 2 & 9 \\
\hline $\begin{array}{l}\text { France } \\
\text { Germany }\end{array}$ & 110 & 138 & 57 & 154 & 138 & 597 \\
\hline Hungary & 0 & 7 & 9 & 32 & 18 & 83 \\
\hline Italy & 7 & 7 & 0 & 8 & 2 & 12 \\
\hline Japan & 55 & 37 & 20 & 74 & 64 & 250 \\
\hline Poland & 24 & 72 & 12 & 60 & 61 & 229 \\
\hline Portugal & 9 & 16 & 7 & 23 & 7 & 62 \\
\hline Spain & 18 & 22 & 13 & 47 & 26 & 126 \\
\hline Switzerland & 2 & 5 & 5 & 11 & 6 & 29 \\
\hline The Netherlands & 11 & 1 & 4 & 7 & 9 & 32 \\
\hline Turkey & 43 & 38 & 41 & 87 & 65 & 274 \\
\hline UK and Ireland & 17 & 24 & 5 & 30 & 19 & 95 \\
\hline US & 65 & 91 & 36 & 119 & 99 & 410 \\
\hline
\end{tabular}

Data: H Lochmüller, manuscript in preparation.

patients across 35 countries, and more will be included in the future as other countries join.

The TREAT-NMD network provides an invaluable research tool for trial readiness (feasibility and recruitment), and to assess standards of care (CARE-NMD), biomarker discovery and validation (BIO-NMD) as well as investigating burden of illness and the natural history and outcomes. The wealth of data that can be obtained is illustrated in Figure 3, which shows the patient numbers for the five most common exon skips across different countries in the TREAT-NMD network in 2010. Selecting a particular exon as an inclusion parameter immediately narrows the cohort, and international collaboration is essential for trial feasibility and recruitment, i.e. to gain sufficient patient numbers to facilitate research.

There are numerous examples of the TREAT-NMD registry being used to address research questions for DMD. As an example, to explore the changing natural history of $\mathrm{DMD}$, registry data for each country in TREAT-NMD were transferred to a central database $(n=6,787) .{ }^{10}$ Only patients with a fully confirmed DMD mutation were included in the final analysis and the data were subject to data cleaning and plausibility checks (H Lochmüller et al., manuscript in preparation). The data showed variation in steroid uptake across different countries, revealing that care standards are being not applied equally worldwide. When the effects of this on outcomes are examined, a negative correlation

\section{Figure 4: Correlation between Steroids and Ambulation from Patient Data Sourced from the TREAT-NMD Registry}
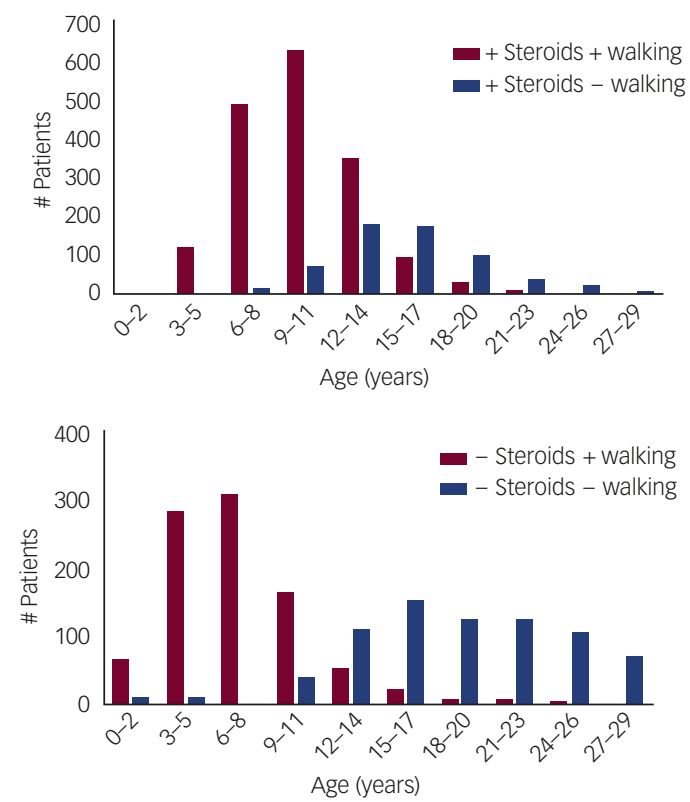

can be discerned between steroid use and lack of ambulation at age 12-17 years (see Figure 4). Steroid users were five times more likely to walk at age 12-17 years than those who did not use steroids. Similarly, an inverse correlation was shown between steroid use and the requirement for scoliosis surgery, whereby those who used steroids were five times less likely to require scoliosis surgery than non-steroid users. No significant effect of steroids on the onset of cardiomyopathy was apparent however.

Expansion is planned for TREAT-NMD registries to assist recruitment for industry-sponsored trials, with new sites and diseases to be included in the Care and Trial Site Registry. In addition, the action plan for 2014 to 2016 includes improved visibility for centres of excellence, data curation to avoid duplication of data entry and collaboration with research networks, patient organisations and with industry to provide feedback to patient organisations and to facilitate post-marketing studies. Regarding the collaboration with industry, post-marketing studies are needed to understand the safety signals and long-term treatment outcomes of new therapies, and may become a requirement by the European Medicines Agency (EMA) and US Food and Drug Administration (FDA). To help fulfil this requirement and to potentially address other long-term research questions, further investment is focussing on setting up a system to allow standardised and more complete data capture (i.e. auditable data, similar to clinical trials) in anticipation of supporting these future needs.
1. Landfeldt $E$, Lindgren $P$, Bell $C F$, et al., The burden of Duchenne muscular dystrophy: An international, crosssectional study, Neurology, 2014;83:529-36.

2. Mendell JR, Shilling C, Leslie ND, et al., Evidence-based path to newborn screening for Duchenne muscular dystrophy, Ann Neurol, 2012;71:304-13.

3. Moat SJ, Bradley DM, Salmon R, et al., Newborn bloodspot screening for Duchenne muscular dystrophy: 21 years experience in Wales (UK), Eur $\mathrm{J}$ Hum Genet, 2013;21:1049-53.

4. Simonds AK, Muntoni F, Heather S, Fielding S, Impact of nasal ventilation on survival in hypercapnic Duchenne muscular dystrophy, Thorax, 1998;53:949-52.

5. Eagle M, Baudouin SV, Chandler C, et al., Survival in Duchenne muscular dystrophy: improvements in life expectancy muscular dystrophy: improvements in life expectancy
since 1967 and the impact of home nocturnal ventilation, since 1967 and the impact of home
Neuromuscul Disord, 2002;12:926-9.

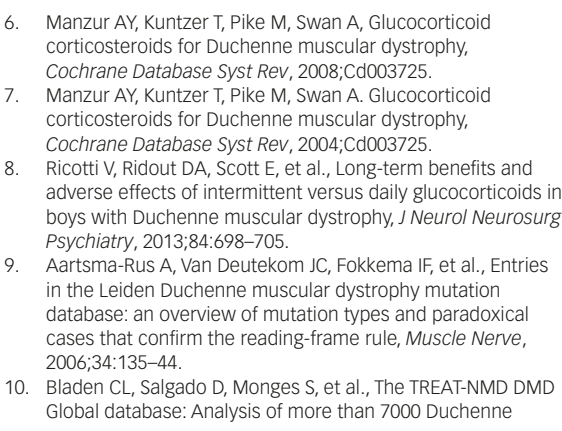

6. Manzur AY, Kuntzer T, Pike M, Swan A, Glucocorticoid corticosteroids for Duchenne muscular dystrophy, Cochrane Database Syst Rev, 2008;Cd003725.

Manzur AY, Kuntzer T, Pike M, Swan A. Glucocorticoid corticosteroids for Duchenne muscular dystrophy, Cochrane Database Syst Rev, 2004;Cd003725.

8. Ricotti V, Ridout DA, Scott E, et al., Long-term benefits and adverse effects of intermittent versus daily glucocorticoids in boys with Duchenne muscular dystrophy, I Neurol Neurosurg Psychiatry, 2013;84:698-705.

9. Aartsma-Rus A, Van Deutekom JC, Fokkema IF, et al., Entries in the Leiden Duchenne muscular dystrophy mutation database: an overview of mutation types and paradoxical cases that confirm the reading-frame rule, Muscle Nerve, 2006:34:135-44.

10. Bladen $C L$, Salgado D, Monges $S$, et al., The TREAT-NMD DMD Global database: Analysis of more than 7000 Duchenne

muscular dystrophy mutations, Hum Mutat, 2015 [Epub ahead of print]

1. Aartsma-Rus A, Dystrophin analysis in clinical trials, Journal of Neuromuscular Diseases, 2014;1 1(1):41-53.

12. Bushby K, Finkel R, Birnkrant DJ, et al., Diagnosis and management of Duchenne muscular dystrophy, part 1: diagnosis, and pharmacological and psychosocial management, Lancet Neurol, 2010;9:77-93.

13. Bushby K, Finkel R, Birnkrant DJ, et al., Diagnosis and management of Duchenne muscular dystrophy, part 2 implementation of multidisciplinary care, Lancet Neurol, 2010;9:177-89.

14. van Ruiten $\mathrm{HJ}$, Straub V, Bushby K, Guglieri M, Improving recognition of Duchenne muscular dystrophy: a retrospective case note review, Arch Dis Child, 2014:99:1074-7.

15. MCDonald CM, Henricson EK, Abresch RT, et al., The 6-minute walk test and other endpoints in Duchenne muscular 
dystrophy: longitudinal natural history observations over 48 weeks from a multicenter study Muscle Nerve, 2013:48:343-56.

16. MCDonald CM. Henricson EK Han It et al. The 6-minute walk test in Duchenne/Becker muscular dystrophy: Iongitudinal observations, Muscle Nerve, 2010;42:966-74.

7. McDonald CM, Henricson EK, Han JJ, et al., The 6-minute walk test as a new outcome measure in Duchenne muscular walk test as a new outcome measure in Duch
dystrophy, Muscle Nerve, 2010;41:500-10

18. Goemans $N$, Klingels $K$, van den Hauwe $M$, et al., Six-minute walk test: reference values and prediction equation in healthy boys aged 5 to 12 years, PloS One, 2013;8:e84120

19. Mazzone ES, Pane M, Sormani MP, et al., 24 month longitudinal data in ambulant boys with Duchenne muscular dystrophy, PloS One, 2013;8:e52512.

20. Pane M, Mazzone ES, Sormani MP, et al., 6 Minute walk test n Duchenne MD patients with different mutations: 12 month changes, Plos One, 2014;9:e83400.

21. Henricson E, Abresch R, Han JJ, et al., The 6-minute walk test and person-reported outcomes in boys with duchenn muscular dystrophy and typically developing controls: longitudinal comparisons and clinically-meaningful changes over one year, PLOS CUrr, 2013:5.

22. MCDonald CM, Henricson EK, Abresch RT, et al., The 6-minute walk test and other clinical endpoints in duchenne muscular dystrophy: reliability, concurrent validity, and minimal clinically important differences from a multicenter study, Muscle Nerve, 2013;48:357-68.

23. Scott E, Eagle M, Mayhew A, et al., Development of a functiona assessment scale for ambulatory boys with Duchenne muscular dystrophy, Physiother Res Int 2012:17:101-9.

24. Mazzone ES, Messina S, Vasco G, et al., Reliability of the North Star Ambulatory Assessment in a multicentric setting, Neuromuscul Disord, 2009:19:458-61.

25. Mayhew A, Cano S, Scott E, et al., Moving towards meaningful measurement: Rasch analysis of the North Star Ambulatory Assessment in Duchenne muscular dystrophy, Dev Med Child Neurol, 2011;53:535-42.

26. Bushby K, Finkel R, Wong B, et al., Ataluren treatment of patients with nonsense mutation dystrophinopathy, Muscle Nerve, 2014; $50: 477-87$

27. Escolar DM, Hache LP, Clemens PR, et al., Randomized; blinded trial of weekend vs daily prednisone in Duchenne muscular dystrophy, Neurology, 2011;77:444-52.

28. De Sanctis R, Pane M, Sivo S, et al., Suitability of North Star Ambulatory Assessment in young boys with Duchenne Ambulatory Assessment in young boys with Duchenne
29. Pane M, Scalise R, Berardinelli A, et al., Early neurodevelopmental assessment in Duchenne muscular dystrophy, Neuromuscul Disord, 2013;23:451-5.

30. Connolly AM, Florence JM, Cradock MM, et al., Motor and cognitive assessment of infants and young boys with Duchenne Muscular Dystrophy: results from the Muscula Dystrophy Association DMD Clinical Research Network, Dystrophy Association DMD Clinical Rese
Neuromuscul Disord, 2013;23:529-39.

31. Mazzone ES Vasco $G$, Palermo $C$ et al., A critical review of functional assessment tools for upper limbs in Duchenne muscular dystrophy, Dev Med Child Neurol, 2012;54:879-85

32. Mayhew A, Mazzone ES, Eagle M, et al., Development of the Performance of the Upper Limb module for Duchenne muscular dystrophy, Dev Med Child Neurol, 2013;55:1038-45.

33. Rodger S, Lochmuller H, Tassoni A, et al., The TREAT-NMD care and trial site registry: an online registry to facilitate clinical research for neuromuscular diseases, Orphanet I Rare Dis, 2013;8:171.

34. Bladen CL, Rafferty K, Straub V, et al., The TREAT-NMD Duchenne muscular dystrophy registries: conception, design, and utilization by industry and academia, Hum Mutat, 2013;34:1449-57 